\title{
Real Time Implementation and Investigation of Wireless Device of Electrical Stimulation for Peripheral Nevers
}

\author{
Mrs. R. Ponni' , S. Manisha², A. Monisha², G. Nandhini², R. Priyatharcini2 \\ ${ }^{1}$ Assistant Professor, ${ }^{2}$ Student \\ 1,2Department of Electronics and Communication Engineering, Kings College of Engineering, Tamil Nadu, India
}

\begin{abstract}
How to cite this paper: Mrs. R. Ponni | S. Manisha | A. Monisha | G. Nandhini | R. Priyatharcini "Real Time Implementation and Investigation of Wireless Device of Electrical Stimulation for Peripheral Nevers" Published in International Journal of Trend in Scientific Research and Development

(ijtsrd), ISSN: 2456-

6470, Volume-3 |

Issue-3, April 2019,

pp.

91-95.

http://www.ijtsrd.co

$\mathrm{m} /$ papers/ijtsrd216

72.pdf

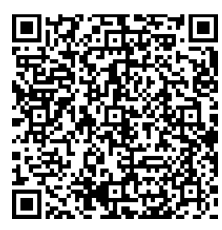

IITSRD21672
\end{abstract}

Copyright (C) 2019 by author(s) and International Journal of Trend in Scientific Research and Development Journal. This is an Open Access article distributed under the terms of the Creative Commons

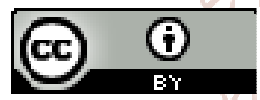
Attribution License (CC BY 4.0) (http://creativecommons.org/licenses/ by $/ 4.0$ )

\section{INTRODUCTION}

In recent years, the average age of the world's population has been increasing, and healthcare monitoring devices have received a lot of attention because they improve the length and quality of people's lives. Healthcare monitoring includes caring for the welfare of every person, which includes early diagnosis of diseases, real-time monitoring of the effects of treatment, therapy, and the general monitoring of the conditions of people's health. As a result, wearable electronic devices are receiving greater attention because of their facile interaction with the human body, such as monitoring heart rate, wrist pulse, motion, blood pressure, intraocular pressure, and other health-related conditions. Unlike conventional health-monitoring systems (e.g., blood pressure meters), wearable electronic devices are portable, wearable, and they provide real-time, continuous, recorded data related to complex health conditions in a timely manner. Furthermore, in order to maximize the portable and wearable advantages of the wearable devices, energy harvesting devices were integrated instead of rigid and bulk batteries. These characteristics of wearable electronic devices can improve the users' or patients' compliance with medical instructions and medication schedules. Since the wearable devices are wireless, the information they gather can be sent to a central node, such as a cell phone or a microcontroller board that can transmit the information to a medical center and display it on a screen.

According to the World Health Organization (WHO), approximately 15 million people suffer strokes and chronic pains worldwide each year. Strokes and chronic pains is a very broad term and include a variety of different types of diseases involving the blood vessels that supply the brain. Treatment depends on the type of stroke and the location of the blood vessels involved. So we develop a Non-Invasive artificial stimulation for those who are unfortunate enough to move their hands due to paralyze attack or coma.

\section{METHODS AND MATERIAL}

Segmentation of Brain Stroke Image by Abdulrahman Alhawaimil talks about Stroke is the 4th leading cause of death in the US, with one person dying every 4 minutes as a result. Approximately 800,000 people have a stroke each year; about one every 4 seconds. Strokes occur due to problems with the blood supply to the brain; either the blood supply is blocked or a blood vessel within the brain ruptures. Due to the increase in misdiagnosis in medical for example 
misdiagnosis of stroke in brain. This Led to make correct medical diagnosis through many ways. However, it is not easy to correctly diagnose the stroke. In this paper our aim to present a foundational approach to assisting physicians when making medical diagnose for brain stroke. Brain stroke has been detected using image segmentation. The researcher use Matlab image processing software to analyze brain stroke image captured from magnetic resonance imaging (MRI).

The Robot that Learns from the Therapist How to Assist Stroke Patients by M. D. Kostic, M. D. Popovic, D. B. Popovic talks about Results from clinical studies suggest that assisted training is beneficial for the recovery of functioning in patients with stroke and other central nervous system injuries. The training consists of the repetition of movements, which change the excitability of the brain, and due to cortical plasticity have carry-over effects. We are developing a 3D arm assistant that interfaces the patient at the hand/wrist. The development addresses three major issues: (1) the selection of the tasks that are appropriate for the training based on the level of motor abilities (2) the design of the visual feedback that enhances the motivation to train, and (3) the assessment of the performance. Therefore, our design integrates the new 3D robot assistant, various gaming based visual feedback, and software that acquires data on-line from sensors (position of the hand and force between the robot and the hand). The major novelties that the 3D arm assistant brings are the following: an automatic method of capturing movements presented by the therapist (expert), the use of the probabilistic movement representation for control of the robot, the incorporation of simple gaming with adjustable levels of difficulty, and finally, the assessment of differences between the achieved and target movements (kinematics) and interface force measured by a special handle with multiple sensors. The components of the new arm assistant in 2D have been tested and proved to work effectively in the clinical trials with stroke patients.

Detection and Segmentation of Ischemic Stroke Using Textural Analysis on Brain CT Images by Alyaa Hussein Ali, Shahad Imad Abdulsalam, Ihssan Subhi Nema talks about The detection of brain strokes from Computed Tomography CT images needs convenient processing techniques starting from image enhancement to qualify the brain image by isolation process, region growing and logical operators (OR and AND). These methods with the help of the simplest segmentation process, which is the thresholding process, are used to extract a stroke region from the CT image of the brain. The median filter is applied to remove the noise from the image. The statistical features calculated using firstorder histogram was utilized in the detection of the stroke region.

Machines to support motor rehabilitation after stroke: 10 years of experience in Berlin by Stefan Hesse, MD Henning Schmidt talks about The group at Klinik Berlin/Charite University Hospital in Berlin, Germany, began studies to promote motor recovery after stroke in the early 1990s. Following the introduction of treadmill training with partial body-weight support, the group designed and electromechanical gait trainer, GT I, based on movable foot plates that relieve therapist effort (e.g., when assisting the movement of the paretic limbs) and intensify patient gait training (GT). Preliminary results of a recent multicenter trial of 155 acute stroke patients showed that the GT I effectively promote gait ability and competence in activities of daily living. The experimental group received $20 \mathrm{~min}$ of GT and 25 min of physiotherapy (PT) and the control group received $45 \mathrm{~min}$ of PT every day for 4 weeks. The laboratory's next step was the HapticWalker, a robotic walking simulator with freely programmable foot plates so that patients can, for example, additionally train for stair climbing and perturbations. The foot plates can be operated in full guidance or compliance control modes, thus reducing foot support according to the patient's learning success. For the severely affected upper limb, the laboratory's computerized arm trainer, called the Bi-Manu-Track, enables bilateral practice of forearm pronation/supination and wrist flexion/extension. Compared with electrical stimulation of the paretic wrist extensors, acute stroke patients with severe arm paresis $(n=44)$ had significantly more upper-limb muscle strength and control at the end of the 6-week intervention period and at follow-up. The laboratory's most recent and cost-effective development, the Nudelholz, is a purely mechanical device that bilaterally trains the shoulder, elbow, and wrist joints. It is intended for home therapy.

\section{MICROCONTROLLER}

Arduino:

It is an open source computer hardware and software company, project, and user community that designs and manufactures single-board microcontrollers and microcontroller kits for building digital devices and interactive objects that can sense and control objects in the physical world. The project's products are distributed as open-source hardware and software, which are licensed under the GNU Lesser General Public License (LGPL) or the GNU General Public License (GPL), permitting the manufacture of Arduino boards and software distribution by anyone. Arduino boards are available commercially in preassembled form, or as do-it-yourself (DIY) kits.

Arduino board designs use a variety of microprocessors and controllers. The boards are equipped with sets of digital and analog input/output (I/O) pins that may be interfaced to various expansion boards (shields) and other circuits. The boards feature serial communications interfaces, including Universal Serial Bus (USB) on some models, which are also used for loading programs from personal computers. The microcontrollers are typically programmed using a dialect of features from the programming languages $\mathrm{C}$ and $\mathrm{C}++$. In addition to using traditional compiler tool chains, the Arduino project provides an integrated development environment (IDE) based on the Processing language project.

The Arduino project started in 2003 as a program for students at the Interaction Design Institute Ivrea in Ivrea, Italy, aiming to provide a low-cost and easy way for novices and professionals to create devices that interact with their environment using sensors and actuators. Common examples of such devices intended for beginner hobbyists include simple robots, thermostats, and motion detectors. Arduino microcontrollers are pre-programmed with a boot loader that simplifies uploading of programs to the onchip flash memory. The default bootloader of the Arduino UNO is the optiboot bootloader.[28] Boards are loaded with program code via a serial connection to another computer. Some serial Arduino boards contain a level shifter circuit to convert between RS-232 logic levels and transistor- 
transistor logic (TTL) level signals. Current Arduino boards are programmed via Universal Serial Bus (USB), implemented using USB-to-serial adapter chips such as the FTDI FT232. Some boards, such as later-model Uno boards, substitute the FTDI chip with a separate AVR chip containing USB-to-serial firmware, which is reprogrammable via its own ICSP header. Other variants, such as the Arduino Mini and the unofficial Boarduino, use a detachable USB-toserial adapter board or cable, Bluetooth or other methods. When used with traditional microcontroller tools, instead of the Arduino IDE, standard AVR in-system programming (ISP) programming is used.

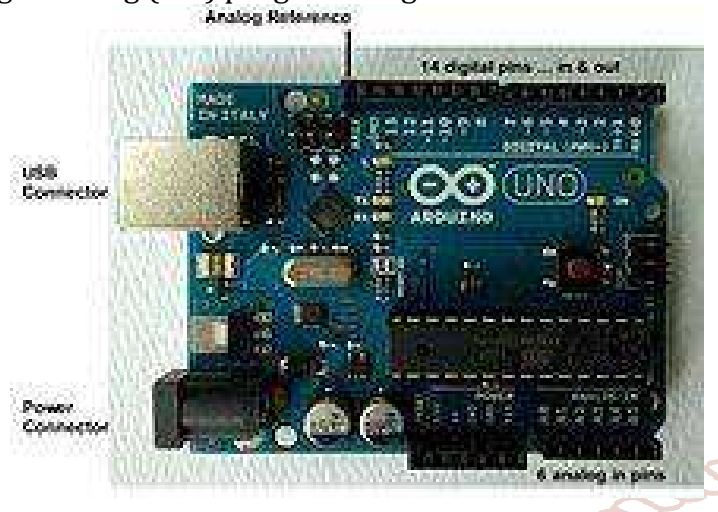

An official Arduino Uno R2 with descriptions of the I/O locations

\section{EMG SENSOR}

Electromyography (EMG) enables the translation of this surface into electrical signals, enabling them to be used in a wide array of applications. Our sensor is especially designed for surface EMG, and works both with pre-gelled and most types of dry electrodes. The bipolar configuration is ideal for low-noise data acquisition, and the raw data output enables it to be used for human-computer interaction and biomedical. Electromyography (EMG) is an electro diagnostic medicine technique for evaluating and recording the electrical activity produced by skeletal muscles.To conduct EMGs, the red and green leads need to be placed on electrodes that are attached to the muscle of interest. The two leads are interchangeable for EMGs. Place these leads along the length of the muscle. The black lead serves as a ground. It should not be placed on the muscle of interest. Two electrode tabs should be placed on the ventral forearm, 5 and $10 \mathrm{~cm}$ from the medial epicondyle with the ground electrode on the upper arm. Alternatively, the ground electrode can be placed on the wrist of the adjacent arm to minimize movement artifacts.

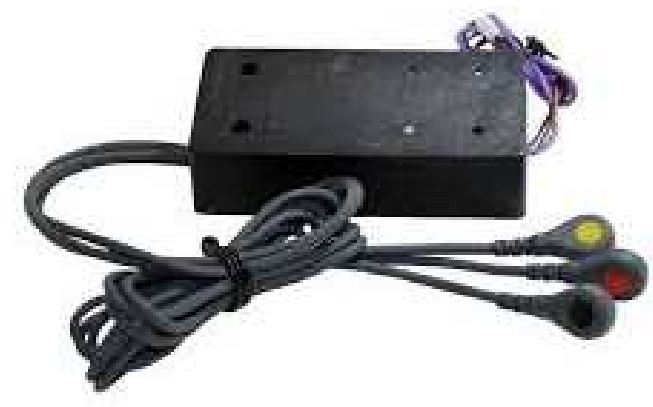

\section{NERVE SIMULATOR SENSOR}

We have attempted to quantify the performance of natural versus artificial sensors when used in a closed-loop functional electrical stimulation system. Peroneal nerve stimulation was applied during gait to a multiple sclerosis subject with a drop foot. Stimulation was applied only during the swing phase to provide artificially induced dorsiflexion of the foot. Detection of the onset of the stance phase was accomplished using a standard heel contact switch mounted inside the subject's shoe (the artificial sensor) and using processed nerve signals derived from an implanted nervecuff electrode (the natural sensor). A detection percentage of at least $85 \%$ was achieved using the afferent nerve signal information only. It's an electrifying time to be in neuroscience. Using implanted devices that send pulses of electricity through the nervous system, physicians are learning how to influence the neural systems that control people's bodies and minds. These devices give neurologists new ways to treat patients with a wide range of disorders, including epilepsy, chronic pain, depression, and Parkinson's disease. So far, these stimulators have been one-way devices that deliver a steady sequence of pulses to the nervous system but can't react to changes in the patient's body. Now, at last, medical device companies are coming out with dynamic neural stimulators that have a bit of "brain" themselves.

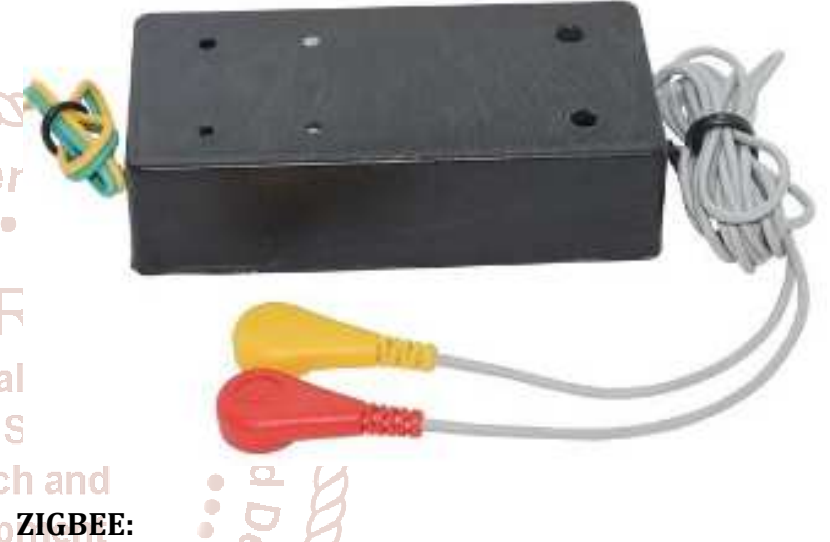

Zigbee is the name of a specification for a suite of high level communication protocols using small, low-power digital radios based on the IEEE 802.15.4 standard for wireless personal area networks (WPANs), such as wireless headphones connecting with cell phones via short-range radio. The technology is intended to be simpler and cheaper than other WPANs, such as Bluetooth. Zigbee is targeted at radio-frequency (RF) applications which require a low data rate, long battery life, and secure networking. Zigbee is an IEEE 802.15.4-based specification for a suite of high-level communication protocols used for wireless networking. It is a wireless technology developed as an open global standard to address the unique needs of low-cost, low-power wireless M2M networks. Zigbee (CC2500) is a low cost true single chip $2.4 \mathrm{GHz}$ transceiver designed for very low power wireless applications. The RF transceiver is integrated with a highly configurable baseband modem.

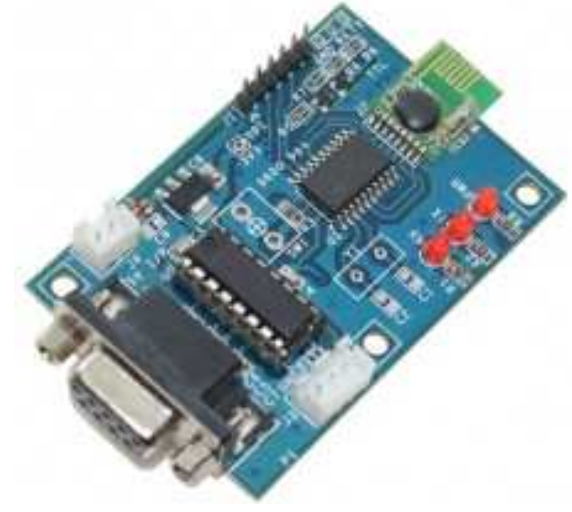




\section{RESULTS AND DISCUSSION}

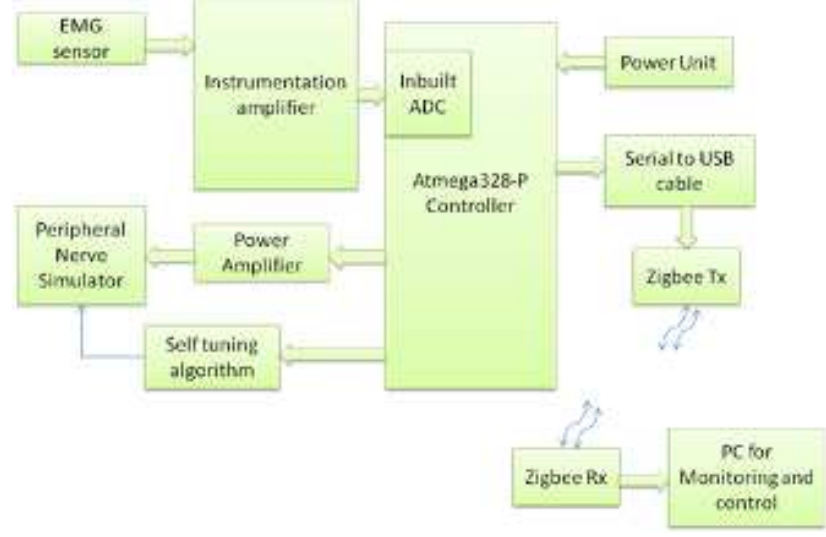

\section{POWER SUPPLY:}

A suitable ready-built mains power supply unit, such as those used to control model trains, will include a transformer. If the unit does not have a fuse or a cut-out on the output of the transformer, you will also need to add a fuse of an appropriate rating. This fuse is in addition to the mains fuse in the unit's plug and is needed to protect the low voltage winding of the transformer and any circuits you connect to it. Although we won't be building the transformer block of our $5 \mathrm{~V}$ regulated power supply.
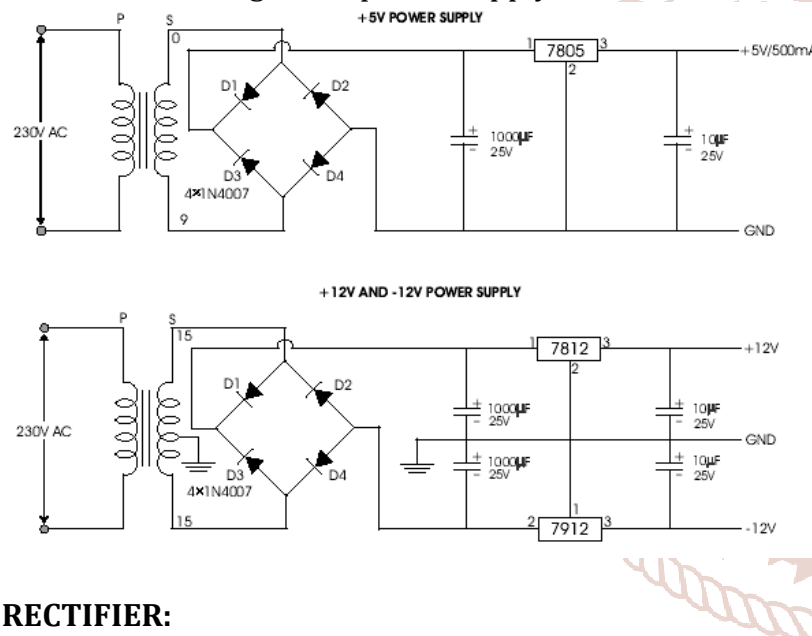

The purpose of a rectifier is to convert an AC waveform into a DC waveform. There are two different rectification circuits, known as 'half-wave' and 'full-wave' rectifiers. Both use components called diodes to convert AC into DC.In the power supply it used bridge rectifier.

\section{BRIDGE RECTIFIER:}

In the rectifier, we wanted our equipment to be turned on and off on the cycle of the AC power. As you can guess we need to have a device that will give us constant power (DC).This is accomplished by using 4 diodes connected in a certain way to make use of both the negative and positive parts of the AC wave.

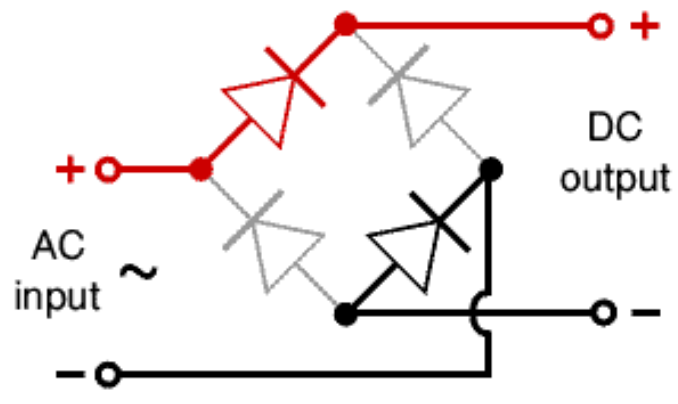

It is very important to make sure the diodes are connected in the right configuration for a Bridge Rectifier to work properly. A Bridge Rectifier provides what is called a "Fullwave varying DC" output and would look like the wave

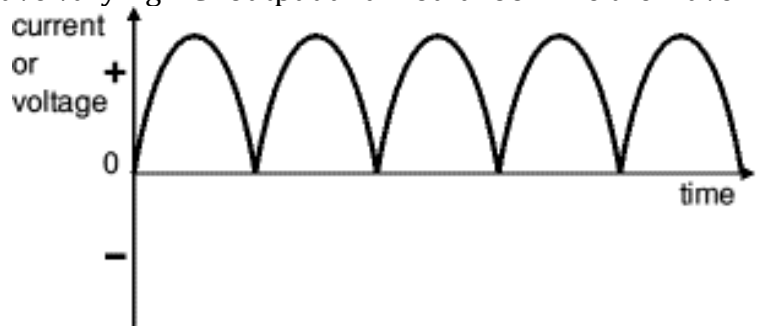

Fig 4.4 Full-wave varying DC

Now before we go any further what is important to remember is that diodes use up.Therefore as voltage has to pass through 2 diodes to complete the circuit on each half cycle the Bridge Rectifier will use up 1.4 Volts of the power provided. Bridge Rectifiers are rated in the following way: Bridge rectifiers are rated by the maximum current they can pass. Also they are rated by the maximum reverse voltage they can withstand (this must be at least three times the supply $\underline{\mathrm{RMS}}$ voltage so the rectifier can withstand the peak voltages). Now that we have a pulsating full-wave signal we now need to flatten or smooth out the signal (straighten to represent a DC signal)

\section{REGULATOR:}

A voltage regulator is designed to automatically maintain a constant voltage level. A voltage regulator may be a simple "feed-forward" design or may include negative feedback control loops. It may use an electromechanical mechanism, or electronic components. Depending on the design, it may be used to regulate one or more AC or DC voltages. Electronic voltage regulators are found in devices such as computer power supplies where they stabilize the DC voltages used by the processor and other elements. In automobile alternators and central power station generator plants, voltage regulators control the output of the plant.

\section{THE 78XX SERIES OF REGULATORS:}

There are many types of regulator IC and each type will have different pin-outs and will need to be connected up slightly differently. Therefore, this article will only look at one of the common ranges of regulator, the $78 x x$ series.

If you are using a regulator after the smoothing block of the power supply, then you should not need to worry about the ripple voltage, since the whole point of using a regulator is to get a stable, accurate, known voltage for your circuits.

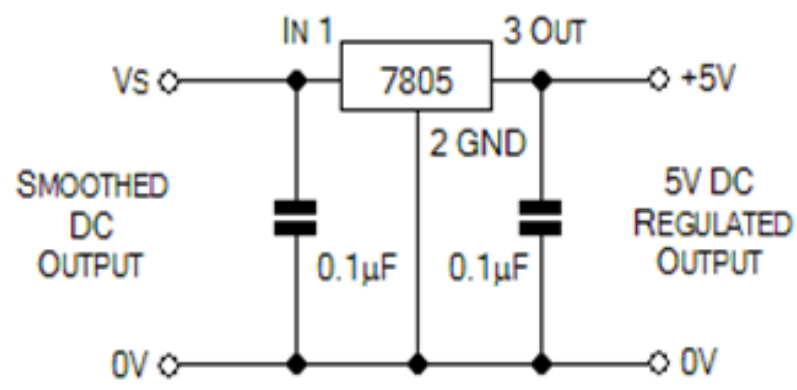

LCD:

LCD (Liquid Crystal Display) screen is an electronic display module and find a wide range of applications. A 16x2 LCD display is very basic module and is very commonly used in 
various devices and circuits. These modules are preferred over seven segments and other multi segment LEDs. The reasons being: LCDs are economical; easily programmable; have no limitation of displaying special \& even custom characters (unlike in seven segments). Each pixel of an LCD typically consists of a layer of molecules aligned between two transparent electrodes

LCD

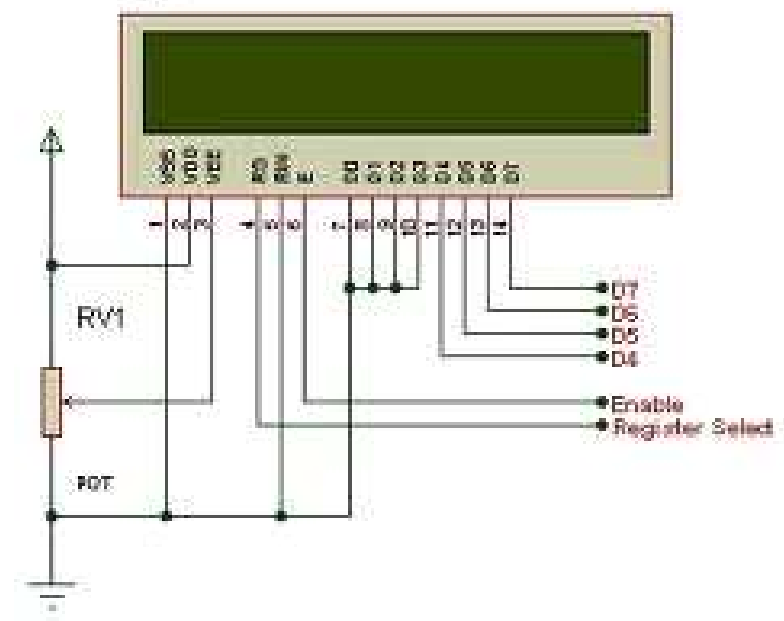

CONCLUSION

An electrical stimulation implant is demonstrated with $>10 \times$ improvement in the depth/volume ratio over existing ICbased neurostimulators. The fully integrated implant measures just $2 \mathrm{~mm} \times 3 \mathrm{~mm} \times 6.5 \mathrm{~mm}(39 \mathrm{~mm} 3,78 \mathrm{mg})$, and operates at a large depth of $10.5 \mathrm{~cm}$ in a tissue phantom. This significant improvement was enabled by the use of ultrasound for wireless powering (at safe intensity levels $3 \times$ below the FDA limit), and a unique co-design strategy for complete operation of the implant during the charging transient of CSTOR. The implant system also implements a robust DL command/data transfer protocol for fully programmable stimulation, and a high compliance voltage $(15 \mathrm{~V})$ stimulator for chronic applications. The stimulator supports biphasic current-controlled stimulation with a wide range of parameters required for electrical stimulation of peripheral nerves. The implant also includes a blue LED which could enable optogenetic stimulation applications in the future. The capability of the implant is demonstrated through in vitro characterizations, and electrical stimulation ex-periments of a sciatic nerve, enabling its use for nextgeneration electroceuticals.

\section{FUTURE ENHANCEMENT}

Future work involves design of a conformal ultrasound TX array for optimization of the end-to-end ultrasonic link efficiency, investigating techniques to mitigate the effect of implant misalignment, and biocompatible packaging of the implant for chronic in vivo applications.

\section{REFERENCES}

[1] K. Famm, B. Litt, K. J. Tracey, E. S. Boyden, and M. Slaoui, "A jump-start for electroceuticals," Nature, vol. 496, pp. 159-161, Apr. 2013.

[2] E. Waltz, "A spark at the periphery," Nature Biotechnol., vol. 34, no. 9, pp. 904-908, Sep. 2016.

[3] B. Bonaz, C. Picq, V. Sinniger, J. F. Mayol, and D. Clarencon, "Vagus nerve stimulation: From epilepsy to the cholinergic anti-inflammatory pathway," Neurogastroenterol. Motility, vol. 25, no. 3, pp. 208221, Mar. 2013.

[4] J. W. Lee, D. Kim, S. Yoo, H. Lee, G.-H. Lee, and Y. Nam, "Emerging neural stimulation technologies for bladder dysfunctions," Int. Neurourol. J., vol. 19, no. 1, pp. 3-11, Mar. 2015.

[5] G. H. Creasey and M. D. Craggs, Functional Electrical Stimulation for Bladder, Bowel, and Sexual Function, 1st ed., vol. 109. Amsterdam, The Netherlands: Elsevier, 2012.

[6] M. Brinton et al., "Electronic enhancement of tear secretion," J. Neural Eng., vol. 13, no. 1, Feb. 2016, Art. no. 016006 .

[7] X. Li, W. A. Serdijn, W. Zheng, Y. Tian, and B. Zhang, "The injectable neurostimulator: An emerging therapeutic device," Trends Biotechnol., vol. 33, no. 7, pp. 388-394, Jul. 2015.

[8] J. H. Schulman, "The feasible FES system: Battery powered BION stimulator," Proc. IEEE, vol. 96, no. 7, pp. 1226-1239, Jul. 2008.

[9] T. J. Foutz, D. M. Ackermann, K. L. Kilgore, and C. C. McIntyre, "Energy efficient neural stimulation: Coupling circuit design and membrane biophysics," PLoS One, vol. 7, no. 12, Dec. 2012, Art. no. e51901.

[10] Y. Shih, T. Shen, and B. Otis, "A $2.3 \mu \mathrm{W}$ wireless intraocular pressure/temperature monitor," IEEE J. Solid-State Circuits, vol. 46, no. 11, pp. 2592-2601, Nov. 2011.

[11] S. Majerus, I. Makovey, H. Zhui, W. Ko, and M. S. Damaser, "Wireless implantable pressure monitor for conditional bladder neuromodulation," in Proc. 2015 IEEE Biomed. Circuits Syst. Conf., Oct. 2015, pp. 1-4.

[12] R. Muller et al., "A minimally invasive 64-channel wireless $\mu$ ECoG implant," IEEE J. Solid-State Circuits, vol. 50, no. 1, pp. 344-359, Jan. 2015. 